\title{
Die moderne Intensivmedizin ist essenziell für das verbesserte Überleben onkologischer Patienten
}

\author{
Norbert Frey
}

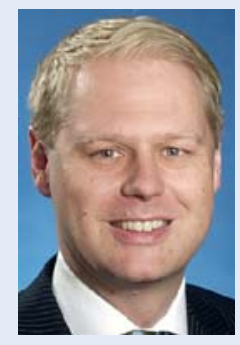

Norbert Frey
Korrespondenzadresse

Prof. Dr. med. Norbert Frey Direktor der Klinik für Innere Medizin III

(Schwerpunkt Kardiologie, Angiologie und Intensivmedizin) Universitätsklinikum Schleswig-Holstein Campus Kiel Schittenhelmstraße 12 24105 Kiel norbert.frey@uk-sh.de

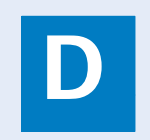

ie onkologische Therapie hat in den letzten Jahren erhebliche Fortschritte gemacht, nicht zuletzt wegen der zunehmenden Verfügbarkeit von „targeted therapies“, wie z.B. monoklonale Antikörper oder Tyrosinkinaseinhibitoren, welche gezielt in den Tumorstoffwechsel eingreifen können. Diese neuartigen Therapien sind häufig schonender als die klassische Chemotherapie und können daher auch bei älteren Patienten mit vorhandenen Komorbiditäten eingesetzt werden. Gleichzeitig verschiebt sich der Altersdurchschnitt onkologisch erkrankter Patienten immer mehr nach oben: So wird für die USA erwartet [1], dass im Jahre 2040 73\% der Krebspatienten älter als 65 Jahre alt sind („silver tsunami“). Auch die „klassische“ Tumortherapie einschließlich Chirurgie und Chemo-/Radiotherapie wird also künftig bei immer älteren Patienten eingesetzt werden. Hieraus ergibt sich, dass Krebspatienten zunehmend bereits andere Komorbiditäten aufweisen, andererseits aber auch durch Komplikationen der Therapie besonders gefährdet sind. Aus allen genannten Aspekten ergibt sich ein weiter steigender Bedarf an intensivmedizinischer Betreuung von Tumorpatienten.

Peter Schellongowski und Mitarbeiter [2] liefern in diesem Kontext einen wichtigen Beitrag in der aktuellen Ausgabe der Intensivmedizin up2date. Zum einen werden relevante tumorspezifische Akuterkrankungen (z.B. Tumorlysesyndrom) diskutiert, zum anderen aber auch spezifische Aspekte bei an Sepsis erkrankten hämatologisch-onkologischen Patienten referiert. Schließlich wird auf die Besonderheiten neutropener bzw. allogen-stammzelltransplantierter Patienten eingegangen.

\section{Ethische Herausforderungen}

Bei vielen schweren Krankheitsbildern immer älterer Patienten steht die Intensivmedizin schon heute vor zunehmenden ethischen Herausforderungen in der Definition der Therapieziele und der Frage, ob immer eine „maximale Intensivmedizin“ zum Einsatz kom- men soll. Dieses gilt in besonderem Maße für onkologische Erkrankungen, z. B. in einer nicht mehr kurativen Situation. Entsprechend definieren die Autoren Kriterien für die Entscheidung „Full code“ vs. „No ICU“. Ein wichtiger Ansatz ist auch der „ICU trial“, d. h. ein zeitlich limitierter Versuch (sinnvollerweise mindestens 3-5 Tage), eine Besserung des akuten Krankheitsbildes mit intensivmedizinischen Maßnahmen zu erreichen. Schon vorher ist ggf. dann eine Absprache sinnvoll, eine Intensivtherapie auch wieder zu deeskalieren. In jedem Fall sollte auch und gerade bei onkologischen Patienten nicht vergessen werden, dass intensivmedizinische Maßnahmen nicht nur das Leben verlängern können, sondern auch zu einer besseren Lebensqualität beitragen, z. B. durch eine nicht-invasive Beatmung bei einem akuten Lungenödem. Dankenswerterweise legen die Autoren einen weiteren Schwerpunkt auf die Darstellung der Ergebnisse intensivmedizinischer Behandlungen von Krebspatienten. Hier zeigt sich, dass in den letzten ca. 20 Jahren erfreuliche Fortschritte erreicht wurden (Verdoppelung des Überlebens). Zu einem therapeutischen Nihilismus besteht also auch auf der Intensivstation kein Anlass. Allerdings verlangt gerade der Tumorpatient eine besonders genaue Abwägung von Möglichkeiten und Grenzen des Einsatzes von Intensivmedizin. Diese Herausforderung lässt sich sicher nur interdisziplinär, ggf. auch unter Einbeziehung von Palliativmedizinern meistern.

\section{Literatur}

1 Bluethmann SM, Mariotto AB, Rowland JH. Anticipating the "Silver Tsunami": Prevalence Trajectories and Comorbidity Burden among Older Cancer Survivors in the United States. Cancer Epidemiol Biomarkers Prev 2016; 25: 1029-1036

2 Schellongowski P, Beutel G, Kochanek M et al. Intensivmedizinische Aspekte bei hämatologischen und onkologischen Erkrankungen. Intensivmedizin up2date 2016; 12: 267- 285 